\title{
PsychoPro 2.0: Using multidimensional scaling to examine the perceptual categorization of race
}

\author{
Otto H. Maclin, Dwight J. Peterson, Cody Hashman, and Nick Flach \\ University of Northern Iowa, Cedar Falls, Iowa
}

\begin{abstract}
Recently, researchers examining cognitive mechanisms involved in the detection of racial markers have been using psychophysics to examine perceptual thresholds delineating race using a program called PsychoPro (MacLin, MacLin, Peterson, Chowdhry, \& Joshi, 2009). PsychoPro allows researchers to collect data using facial stimuli morphed along a racial continuum with psychophysical methodology. PsychoPro 2.0 was updated so researchers could collect paired-comparison data that can then be analyzed using multidimensional scaling (MDS). MDS allows researchers to examine the perceptions of racial boundaries along perceptual dimensions not bounded by the stimulus continuum, by having the observer make paired comparisons to racial stimuli based on their perceived similarity. Results indicate that ratings were made on the basis of at least six different racial dimensions that suggest the emergence of a third race not represented by the original morph starting images. Details of the software, the new paired-comparison feature and its relevance in psychophysical studies in general and racial categorization in particular are presented.
\end{abstract}

In today's political climate, issues surrounding race have become increasingly relevant to discussions of terrorism, crime, and our recent presidential election. Correspondingly, researchers have refocused empirical attention on race to study factors such as skin tone, cross-race identification, prejudice, and racial categorization. For researchers examining race and race-related issues, computer programs that can display and collect participant responses to a variety of facial stimuli are especially useful and are becoming more readily available and user-friendly.

Researchers for the past 40 years have examined a phenomenon known as the cross-race effect (CRE), in which lower accuracy for recognition of other-race faces than for same-race faces is exhibited (Malpass \& Kravitz, 1969; Meissner \& Brigham, 2001). Although robust, the origins of the phenomenon have yet to be isolated. One of the early theories attempting to explain the CRE is the contact hypothesis. According to this theory, increased contact with members of another race should cause a decrease in the CRE, because individuals presumably devote more attention to facial features of other-race individuals with whom contact has increased (Malpass, 1981; Shepherd, 1981). While this and other socially based explanations for the CRE encompass relevant hypotheses that make intuitive sense, other researchers posit that examining cognitive explanations is also important (Levin, 1996; Valentine, 1991).

Some of these cognitive theories assume that deficiencies in encoding, and subsequent recognition for otherrace faces, serve to explain the CRE. One such theory views race as a feature, thus driving the advantages in cat- egorization based on race when an individual is exposed to a member of another race (Levin, 1996). According to Levin, other-race faces are processed on the basis of the presence or absence of certain features specific to a particular race. This is relatively easy, yet results in a deficit for individuating these other-race members from one another.

Another theory is based on the encoding and storage of individual faces in a conceptual $n$-dimensional face space (Valentine, 1991). According to this theory, other-race faces are stored closely together in a multidimensional face space, whereas same-race faces are stored with greater distance between them within this face space (Valentine, 1991). Because of deficiencies for individuating other-race faces, these faces are viewed as closely approximating the prototypical face of that race, and so are stored in close proximity to one another. This increases the likelihood of one's reporting having seen a face before that one may never have actually seen. Same-race faces, however, are individuated with relative ease, and thus, when encoded, are more distal from one another. This results in greater accuracy in recognizing same-race faces (Valentine, 1991). Together, these socially and cognitively based theories provide a wealth of potential explanations for the CRE, but none have yet attempted to integrate the theories into a comprehensive model that could account for much of what is already known and what has yet to be explained.

On the basis of research examining ambiguous-race faces, MacLin and Malpass $(2001,2003)$ found that identical faces are processed differently when a racial marker

O. H. MacLin, otto.maclin@uni.edu 
consisting of hair was used. Specifically, they discovered a perceptual illusion, finding that faces with an AfricanAmerican hair style were perceived as being darker than faces with the same-race hair style (MacLin \& Malpass, 2003). This effect was later replicated by Levin and Banaji (2006). Additionally, MacLin and Malpass (2001) were able to create a cross-race effect by simply manipulating hair style on otherwise identical stimuli. They posited that the racial marker acts as a signal to the brain to process the faces differently. Clearly, if we are to fully understand the cross-race effect, researchers need to study how other-race faces are processed and how racial markers are involved in the process.

Recently, MacLin and MacLin (in press; MacLin, MacLin, Peterson, Chowdhry, \& Joshi, 2009; MacLin et al., 2008) proposed a theory for a cognitive gating mechanism (CGM) for the processing of racial information during face recognition (see Figure 1). According to the theory, once a face is detected in the environment, information is routed either along typical face processing channels (for in-group or same-race faces) or along another processing channel (for out-group or other-race faces). The process for same-race faces is similar to the model presented by Bruce and Young (1986), which posited that once an object is recognized as a face, the next stage is to determine if it is familiar. If the face is familiar, an attempt to retrieve semantic information from long-term memory follows. If semantic information is retrieved, attempted retrieval of a name is the final step (see also Bruce, Burton, \& Hancock, 2007). According to their model, the process is linear and each step must yield a successful outcome before the succeeding step is attempted. A shortcoming of the Bruce and Young model is that it does not account for the processing of other-race faces, nor does it make any distinction between same- and other-race faces.

The MacLins' model is based on data indicating that faces are processed differently when a racial marker is present. Racial markers can be features such as skin tone, hair, or eye color. Once a racial marker is detected, the image of the face is sent to other areas of the brain (see MacLin \& MacLin, in press, for support of this proposition). Unlike same-race faces, which are processed configurally for social reasons - including individuation for later recognition purposes - other-race faces are processed featurally and categorically for action-based responses (e.g., a fight-or-flight response). Unlike same-race faces, which involve accessing long-term memory for the storage and retrieval of semantic information, other-race faces are associated with stereotypes and labels stored in long-term memory (Hugenberg \& Sacco, 2008). These different processes yield different outcomes.

MacLin and MacLin argue that most models explaining the cross-race effect are deficit models, necessarily suggesting a problem with the processing of other-race faces. Malpass and Kravitz (1969) were the first to collect about 40 years' worth of research data on cross-racial recognition, all of which used a recognition-based methodology similar to that in Malpass and Kravitz's original work (Meissner \& Brigham, 2001). We argue here that the deficit is an artifact of the recognition methods used. If Malpass and Kravitz had examined differences in how we classify same- and other-race faces, the outcome would have been entirely different. We would have seen an otherrace advantage, given that people are faster at classifying other-race faces than same-race faces (Levin, 1996). There still remains a cross-race effect, so the basis of this phenomenon will continue to elude us if we insist on examining it using recognition-based tasks; this puts researchers in the position of treating it as a problem or deficit.

From an ecological perspective, one could consider the potential adaptive pressures within the environmental context in which our face-processing mechanisms evolved. Groups avoid (or evaluate) people who look different or have an unusual feature relative to in-group members. Dif-

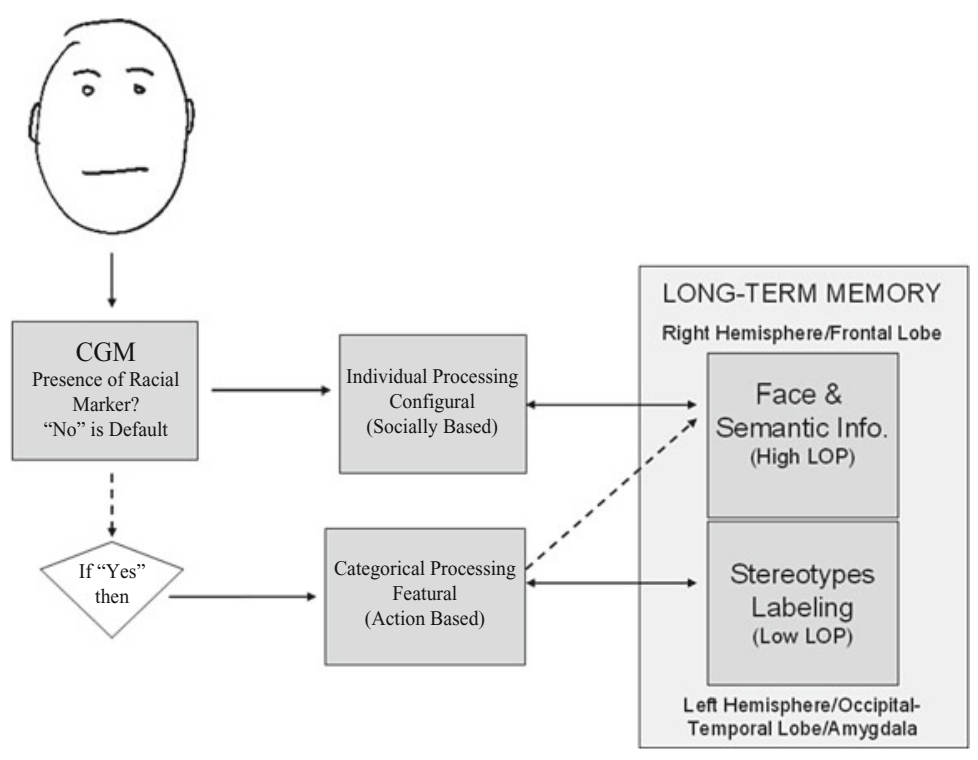

Figure 1. The cognitive gating mechanism (CGM). 
ferentiating between safe, in-group members and potentially threatening out-group members would have been an important, self-protective skill.

We should also note that a large body of research on face recognition has examined differences between configural (holistic) and featural processing (Diamond \& Carey, 1986; Farah, Tanaka, \& Drain, 1995), and has determined that featural processing is inferior to configural processing. Again, most of these studies use recognition-based tests to evaluate configural and featural processing (see Diamond $\&$ Carey, 1986). Studies have found that inverting a samerace face will reduce recognition performance, because inverted faces are processed featurally (Rhodes, Brake, Taylor, \& Tan, 1989; Tanaka \& Farah, 1993; Yin, 1969), whereas inverting an other-race face will not, because upright other-race faces are already processed featurally (MacLin, Van Sickler, MacLin, \& Li, 2004). MacLin and MacLin (in press) argue that inverted faces are processed as part of an out-group, using the same cognitive process used for other-race faces. This perspective provides a more parsimonious explanation than having a separate cognitive mechanism for upside-down faces. From an evolutionary perspective, featural processing must be functional, or it would have been selected out of our cognitive repertoire long ago. From this perspective, the MacLin and MacLin model is an optimal processing model that allows us to ask what the benefits are of featural processing rather than a deficit model that leads us only to ask why we are bad at recognizing other-race faces.

To examine experimentally this evolution-based theory, a computer program called PsychoPro was developed using the Visual Basic 2005 programming language (MacLin \& MacLin, 2007; MacLin et al., 2009). The original program allows for various psychophysical methodologies to be used to conduct experiments examining racial stimuli (for a review of psychophysics, see Snodgrass, 1975). Because psychophysics examines the relationship between physical properties of stimuli and observer perceptions of such stimuli, using these methodologies to examine racial stimuli is especially useful. Racial stimulus continua can be created using morphing programs such as FantaMorph 3.0 (Abrosoft, 2008) to morph two faces of different races together. With PsychoPro, these continua can be presented to participants using various psychophysical methodologies.

PsychoPro makes it easy to use psychophysical methods to examine racial boundaries similar to research by Webster, Kaping, Mizokami, and Duhamel (2004). Webster et al. examined racial boundaries by using a Japanese face and a Caucasian face to create a morphed racial continuum. The faces were converted to grayscale and cropped to remove exterior features such as hair and ears.
Observers indicated the perceived race by clicking one of two buttons indicating race (Japanese or Caucasian) in a two-alternative forced choice (2AFC) task. The researchers were then able to use a staircase method that, on the basis of observer responses, increased or decreased stimulus values along the continuum; each time the stimulus values reversed, the reversal point was measured. Once 12 reversals occurred, the session was completed, with the average of the 12 reversals indicating the racial threshold. Webster et al. found a difference between racial threshold values for Asian students relative to Caucasian students.

The research presented here demonstrates the ability to use PsychoPro to examine racial boundaries using MDS. We present a new addition to PsychoPro (MacLin et al., 2009) that allows the researcher to collect similarity ratings that can be used for analysis with the multidimensional scaling procedure (Kruskal \& Wish, 1978). Rather than being limited to evaluating racial classification along the single dimension of the morph values $(0 \%$ to $100 \%)$, the MDS analysis allows us to examine racial boundaries on $n$-dimensions.

\section{EXPERIMENT 1}

\section{Method}

Observers. Data were collected from 5 trained observers enrolled in a course on Visual Basic programming at the University of Northern Iowa.

Materials. A stimulus continuum of 51 faces was created using FantaMorph (Abrosoft, 2008) with an African-American face as the beginning face, located at the $0 \%$ point on the stimulus continuum, and a Caucasian face as the terminating face, located at the $100 \%$ point on the stimulus continuum. From these, 11 faces were selected. Each face represented a $10 \%$ change along the facial continuum, which included the original African-American parent face $(0 \%)$ and the original Caucasian parent face $(100 \%)$ (see Figure 2$)$. From these, 55 face-pair combinations resulted. (Note that pairs of identical faces were not used.)

Apparatus. A new module designed to present face pairs and collect ratings using a Likert-type scale was created to use with PsychoPro 2.0, an updated version of PsychoPro. With PsychoPro 2.0, the researcher can select from psychophysical methods (constant stimuli, method of adjustment, staircase procedure, lateralization, and MDS); most versions allow for the use of adaptation as an additional feature. For this study, the MDS option was selected. The user interface allows the researcher to indicate the number of facial stimuli desired. Although we chose 11 faces to keep the number of face-pair ratings manageable, the program can present a virtually unlimited number of face pairs. The researcher then indicates the directory path and the file extension for the stimuli. So the file names do not have to be entered individually, the image files must be numbered sequentially with the first image in the sequence named "1.bmp" and with the last face named "11.bmp." Instructions to the participant are read in from text files created by the researcher and are displayed on two separate forms.

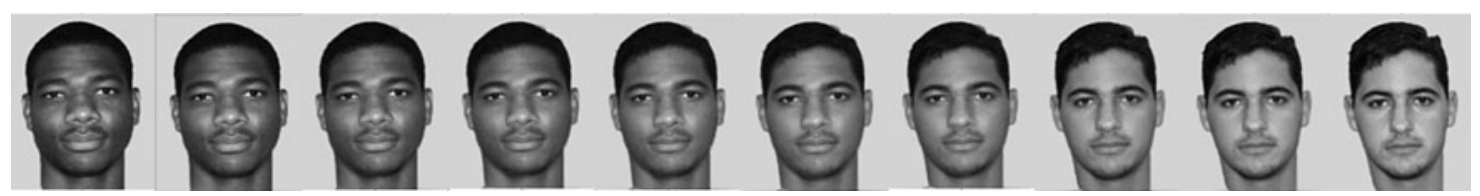

Figure 2. Face stimuli. 
Once the stimulus information and the instructions are read in, the program calculates all possible face pairs and randomizes their presentation (see Figure 3 ). Images are displayed (2 in. $\times$ $3.5 \mathrm{in}$.) with the left/right position of the face images randomized for each presentation to avoid side bias. The observer will use "Continue" buttons to move through the instructions and will be instructed to use the keyboard to enter responses. Faces remain displayed until terminated by an observer's response. Responses are recorded to a comma-delimited text file along with the face-pair names. Once all stimuli are presented, the observer receives final instructions. PsychoPro 2.0 closes any remaining open files, then terminates the run.

Procedure. The observers in this study were aware of the intended nature of the study and the working of the PsychoPro 2.0 program, since they were involved in creating and programming the module. Observers completed the task by themselves on a PC computer. Once all 55 stimuli were presented, the program was terminated.

\section{RESULTS AND DISCUSSION}

Data were arranged into similarity matrices and analyzed together using ALSCAL, a computer software program used to conduct multidimensional scaling (MDS) analyses (see Young \& Lewyckyj, 1979). MDS analyses can easily be computed using statistical software packages such as SPSS, which is equipped with ALSCAL software (for a review of MDS, see Kruskal \& Wish, 1978).

Six and fewer models were requested to determine the most parsimonious model. The optimal model yields low stress and high $r$-squares. Stress and $r$-square values are reported for the 6- to 2-D models successively (.09-.79, $.10-.79, .14-.77, .15-.78$, and .19-.79, respectively). On the basis of these results, we chose a 2-D model, since it explained the variance with fewer dimensions without a loss of $r$-square and with minimal increase in stress.

Using the values from the 2-D model, a stepwise regression was conducted using the percentage morph $(0 \%$ to $100 \%$ ) as the independent variable. Only Dimension 1 contributed to the regression equation $[F(1,10)=90.53$, $p<.001)]$ with an $r$-square of .91 , indicating that faces were grouped according to their systematic change along the stimulus continuum. Correlational analysis indicates that Dimension 2 alone does not correlate significantly to the morph continuum, nor does it correlate with Dimension 1.

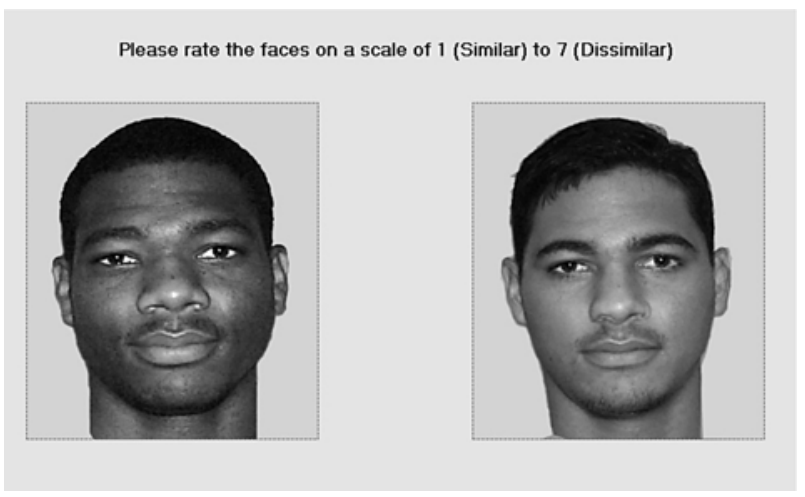

Figure 3. Paired comparison interface.
In order to provide a closer analysis into what is happening with Dimension 2, we conducted a separate analysis of similarity from 1 of the 5 participants. Additionally, we had that observer indicate racial thresholds by conducting a method of constant stimuli with both ascending and descending trials, and superimposed the stimulus coordinates over the matching data ratings.

\section{EXPERIMENT 2}

\section{Method}

Observer. We selected 1 observer randomly from Experiment 1.

Materials. Faces were obtained from the same facial continuum used in Experiment 1, but all 51 faces were used to represent the face continuum, with each representing a $2 \%$ change.

Apparatus. PsychPro 2.0 was used to conduct two sessions of the method of constant stimuli. With descending trials we asked, "Is this face Caucasian?" with "Yes" and "No" as possible responses. We then conducted ascending trials asking "Is this face AfricanAmerican?" with "Yes" and "No" as possible responses. We refer to these as ascending and descending trials, because the observer is forced to use one end of the continuum as a referent, in a similar fashion to traditional ascending and descending trials that ask "Is the light on?" and "Is the light off?" Each set of trials has been known to define a different threshold point. It should be noted that Webster et al. (2004) used a task wherein faces had to be classified as either Asian or Caucasian. Using the ascending and descending trials in Experiment 2 allows for the possibility of faces to be classified as either African-American or Caucasian, and also allows for faces perceived as being of neither race to fall in between the two categories. The benefit of the method of constant stimuli is that it allows us to view the threshold not as a fixed value but as a probability of being categorized by the race in question, forming in some cases an S-shaped curve.

PsychoPro 2.0 can be obtained by contacting the first author.

Procedure. The observer was presented with a block of 51 faces along the racial continuum. Faces were presented in random order. The observer was asked, "Is this face Caucasian?" and provided with a "Yes" and "No" button on which to register a response. Once the observer made a button response, a visual mask appeared for $50 \mathrm{msec}$ to retard the visual afterimage, then was replaced with a new face to categorize. Once all 51 faces were categorized, a second block of faces was presented. This procedure was repeated until 10 blocks of faces were presented. After a break of approximately $3 \mathrm{hr}$, the observer repeated the trials responding to the question "Is this face African-American?" with "Yes" or "No" as the possible response options.

\section{RESULTS AND DISCUSSION}

Data were analyzed separately for the ascending and descending trials. Each face was classified 10 times. If a "No" response was made, a value of 1 was given to that particular face. Responses were averaged, with faces receiving a maximum value of 1 to a minimum value of 0 . As can be seen in Figure 4, faces in the ascending trials (dashed lines) were classified as African-American in all 10 trials up until the $38 \%$ morph, and as not AfricanAmerican in all trials at the $48 \%$ morph. Faces in the descending trials (solid lines) were Caucasian $100 \%$ of the time down to the $68 \%$ morph, and classified as not Caucasian $100 \%$ of the time at the $50 \%$ morph. As such, faces to the left of the dotted line were classified as AfricanAmerican, and faces to the right of the dashed line as 
Observer 1: Constant Stimuli Data

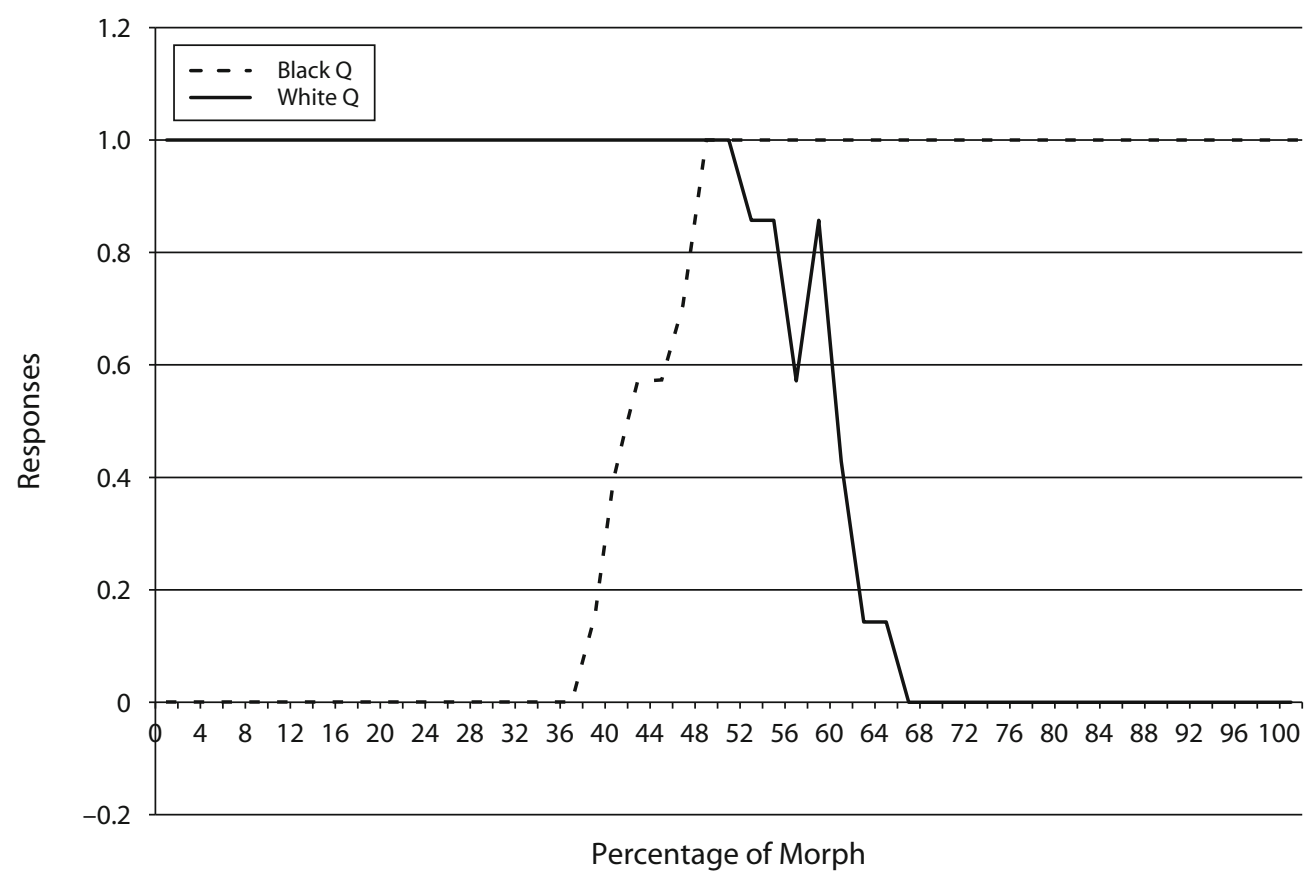

Figure 4. Constant stimuli data.

Caucasian. Faces between the dashed and solid lines were never rated as African-American or Caucasian. MacLin and Malpass (2001) would have referred to these faces as being ambiguous as to race; however, on closer inspection, the faces in the center appear to be South Asian. What seems to be happening is that a new emergent race seems to be appearing at the center of the continuum. We have since investigated this phenomenon, using faces from 40 face continua as stimuli and using a 3AFC task "AfricanAmerican," "Hispanic," and "Caucasian" in a yet unpublished study, and found that about a third of the faces created on an African-American-Caucasian continuum are rated as Hispanic when a $3 \mathrm{AFC}$ paradigm is used.

When the faces from a 2-D MDS solution (see Figure 5) for that observer are superimposed onto the graphical representation of the data, it can be seen that faces comprise three clusters or categories along the stimulus morph dimension (Dimension 1; see Figure 6). The four faces in the left cluster are clearly African-American; the four in the right, clearly Caucasian. The exact race of the faces in the center cluster may be debatable, but they clearly form some new emergent race that is representative of neither the African-American nor the Caucasian parent faces.

\section{GENERAL DISCUSSION}

We have developed a computer program (PsychoPro; MacLin et al., 2009) to evaluate the perception of race within faces generated along a mathematical continuum using morphed stimuli. The program was recently updated to include a module that collects similarity ratings to be analyzed using MDS (PsychoPro 2.0).
On the basis of the two studies presented here, it was demonstrated that, although faces can be created along a mathematical continuum, they are not perceived along that stimulus continuum in the same manner as an ideal observer might perceive them (Joshi et al., 2008). Indeed, on the basis of the MDS solutions, these faces are perceived categorically, with those perceived as being of the race of the parent face located on that end of the continuum. Faces in the middle of the continuum, once believed to be racially ambiguous, are actually perceived as a third emergent race. Evidence from research presently being done in our lab further supports the "emerging race" phenomenon.

Additionally, a second yet undefined dimension (perhaps skin tone or hair length) was present in the 2-D MDS solution. To fully understand the categorization process, we need to know why and how these emergent properties come about. It is important to note that many studies, such as that done by Webster et al. (2004), do not report the emergence of a third race. We believe that they found this as a result of leaving the racial markers intact. Many researchers have resorted to using cropped faces (sometimes referred to as "floating faces") to eliminate problems associated with hair; however, if hair is an important racial marker, and if it is removed from the face, what aspect of race are we studying? Additionally, some researchers (e.g., Webster et al., 2004) control for skin tone by changing the faces to grayscale, removing another important racial marker. With hair and skin tone removed, observers were required to discriminate race on the basis of lesser facial features (perhaps eye shape). Given our findings, we believe that perception of an emergent race occurs as a result of the skin tone and the hair acting together to create a new race: one having dark 
Observer 1: Two-Dimensional Solution

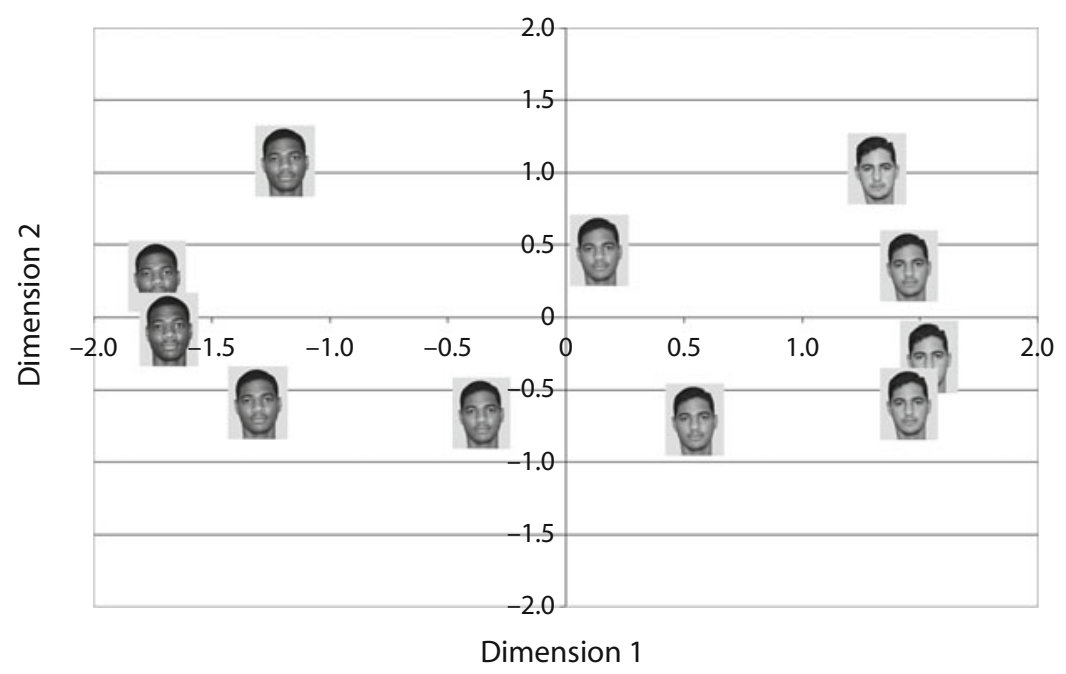

Figure 5. Two-dimensional solution.

skin with straight black hair. In accord with the MacLin and MacLin optimal processing model, the faces with this combination of racial markers are detected as other-race faces and processed differently from same-race faces. Stereotype information and racial labels are then accessed from longterm memory, and perceptual processes are left to interpret race. Within the possible subset of other-race faces that might have dark skin and straight hair (Hispanic, Native American Indian, South Asian, Fiji Islander, etc.), the faces located between the $40 \%$ to $68 \%$ region along the morphed continuum appeared to be most like South Asian.

During 40 years of examining the cross-race effect, no specific model has yet emerged to successfully explain why people of one race have difficulty recognizing peo- ple of another race. This may be because researchers have been examining the cross-race effect using recognitionbased tasks. Any explanation of the effect based on recognition data will likely yield a deficit explanation of why we are bad at recognizing people of other races. An optimal processing model such as that proposed by MacLin and MacLin (in press; MacLin et al., 2008) emerges when the question instead becomes, "How do we process faces of another race compared to same-race faces?" Although we have not fully uncovered the basis for the cross-race effect, nor do we expect to, we believe that it will be various psychophysical methodologies that will allow us to explore the phenomenon, not recognition-based methodologies that only serve to highlight deficit explanations.

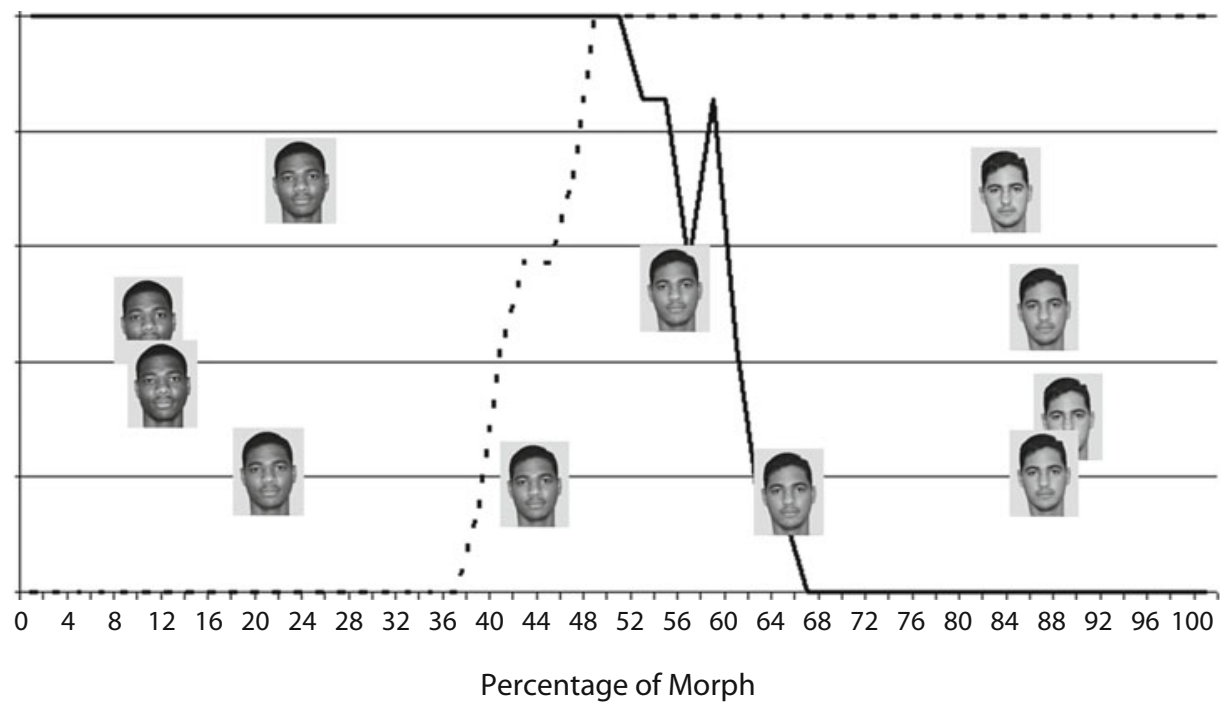

Figure 6. The categorization of faces. 


\section{AUTHOR NOTE}

Correspondence concerning this article should be addressed to $\mathrm{O}$. H. MacLin, Institute for Psychological Science, Department of Psychology, University of Northern Iowa, Cedar Falls, IA 50614 (e-mail: otto .maclin@uni.edu).

\section{REFERENCES}

Aвrosoft (2008). Abrosoft FantaMorph. Retrieved October 29, 2008, from www.fantamorph.com.

Bruce, V., Burton, M., \& Hancock, P. (2007). Remembering faces. In J. D. Read, M. P. Toglia, R. C. L. Lindsay, \& D. F. Ross (Eds.), The handbook of eyewitness psychology: Vol II. Memory for people (pp. 87-100). Mahwah, NJ: Erlbaum.

BRUCE, V., \& Young, A. (1986). Understanding face recognition. British Journal of Psychology, 7, 305-327.

DiAmond, R., \& CAREY, S. (1986). Why faces are and are not special: An effect of expertise. Journal of Experimental Psychology: General, $\mathbf{1 1 5}, 107-117$

Farah, M. J., Tanaka, J. W., \& Drain, H. M. (1995). What causes the face inversion effect? Journal of Experimental Psychology: Human Perception \& Performance, 21, 628-634.

Hugenberg, K., \& SACCO, D. F. (2008). Social categorization and stereotyping: How social categorization biases person perception and face memory. Social \& Personality Psychology Compass, 2, 1052-1072.

Joshi, P., Peterson, D. J., Lane, E., Leslie, D., Maclin, M. K., \& MacLin, O. H. (2008, March). Perceptual discontinuities in the perception of other-race faces: Implications for examining the cross-race effect. Poster presented at the American Psychology-Law Society Annual Conference, Jacksonville, FL.

KrUSKAL, J. B., \& Wish, M. (1978). Multidimensional scaling. Beverly Hills: Sage.

LEvin, D. T. (1996). Classifying faces by race: The structure of face categories. Journal of Experimental Psychology: Learning, Memory, \& Cognition, 22, 1364-1382

Levin, D. T., \& BanaJI, M. R. (2006). Distortions in the perceived lightness of faces: The role of race categories. Journal of Experimental Psychology: General, 135, 501-512.

MacLin, O. H., \& MacLin, M. K. (2007, November). Using PsychoPro to examine social psychological phenomena. Paper presented at the 37th Annual Meeting of the Society for Computers in Psychology, Long Beach, CA.

MacLin, O. H., \& MacLin, M. K. (in press). The role of racial markers in race perception and racial categorization. In R. Adams, N. Ambady, K. Nakayama, \& S. Shimojo (Eds.), People watching: The social ecology of visual perception. New York: Oxford University Press.

Maclin, O. H., Maclin, M. K., Peterson, D., Chowdhry, O., \&
JosHI, P. (2009). Social psychophysics: Using psychophysics to answer "social" questions with PsychoPro. Behavior Research Methods, 41, 623-632.

Maclin, O. H., Maclin, M. K., Peterson, D., Joshi, P., Stone, B., Stewart, J., ET AL. (2008, March). Cross-racial identification and classification: A cognitive gating mechanism for faces. Paper presented at the Cross-Racial Identification Symposium at the 2008 American Psychology-Law Society Annual Conference, Jacksonville, FL.

MacLin, O. H., \& MaLPass, R. S. (2001). Racial categorization of faces: The ambiguous race face effect. Psychology, Public Policy, \& Law, 7, 98-118.

MacLin, O. H., \& Malpass, R. S. (2003). The ambiguous-race face illusion. Perception, 32, 249-252.

MacLin, O. H., Van Sickler, B. R., MacLin, M. K., \& Li, A. (2004). A re-examination of the cross-race effect: The role of race, inversion, and basketball trivia. North American Journal of Psychology, 6, 189-204.

Malpass, R. S. (1981). Training in face recognition. In G. M. Davies, H. Ellis, \& J. Shepherd (Eds.), Perceiving and remembering faces (pp. 271-285). New York: Academic Press.

Malpass, R. S., \& Kravitz, J. (1969). Recognition for faces of own and other race. Journal of Personality \& Social Psychology, 13, 330-334

Meissner, C. A., \& Brigham, J. C. (2001). Thirty years of investigating the own-race bias in memory for faces: A meta-analytic review. Psychology, Public Policy, \& Law, 7, 3-35.

Rhodes, G., Brake, S., TAYlor, K., \& TAN, S. (1989). Expertise and configural coding in face recognition. British Journal of Psychology, 80,313-331.

SHEPHERD, J. W. (1981). Social factors in face recognition. In G. M. Davies, H. Ellis, \& J. Shepherd (Eds.), Perceiving and remembering faces (pp. 55-79). New York: Academic Press.

SNodgrass, J. G. (1975). Psychophysics. In B. Scharf(Ed.), Experimental sensory psychology (pp. 17-67). Glenview, IL: Scott, Foresman.

TANAKa, J. W., \& FAraH, M. J. (1993). Parts and wholes in face recognition. Quarterly Journal of Experimental Psychology, 46A, 225-245.

VAlentine, T. (1991). A unified account of the effects of distinctiveness, inversion, and race on face recognition. Quarterly Journal of Experimental Psychology, 43A, 161-204.

Webster, M. A., Kaping, D., Mizokami, Y., \& Duhamel, P. (2004). Adaptation to natural facial categories. Nature, 428, 557-561.

YIN, R. K. (1969). Looking at upside-down faces. Journal of Experimental Psychology, 81, 141-145.

Young, F. W., \& LEWYCKYJ, R. (1979). ALSCAL-4 user's guide. Chapel Hill, NC: Data Analysis and Theory Associates.

(Manuscript received November 26, 2008; revision accepted for publication February 19, 2009.) 